\title{
Docentes, alumnos y la relación con el saber. Reflexiones en torno a los procesos de subjetividad en una escuela pública de San Luis, Argentina
}

\author{
Teachers, students and the relation to knowledge. \\ Reflexions around the subjectivity processes \\ in a public school in San Luis, Argentina
}

María Guadalupe García ${ }^{1}$

\begin{abstract}
Resumen: El objetivo de este trabajo es analizar los procesos de producción de subjetividad en torno al saber y la relación con este en una escuela de gestión estatal -con características urbano marginal-, a través de las voces de sus directivos y docentes, en cuanto describen a sus alumnos y a sus padres; en el marco de los resultados encontrados en una experiencia de intervención realizada por un equipo de investigación de la Universidad Nacional de San Luis. A partir del análisis de las entrevistas a los directivos y los docentes, desde una metodología cualitativa, se busca interpelar los sentidos y las condiciones de aprendizaje de los alumnos. Se interpreta que, en sus discursos acerca de cómo describen a los estudiantes y a sus propias prácticas de enseñanza, se establecen procesos subjetivantes que los vinculan con un conocimiento especializado, independiente de los modos de relación que ellos establezcan con el mundo, con su entorno, con sus intereses y su propia cultura; entendiéndolos como "sujetos epistémicos" y no como "sujetos del saber".
\end{abstract}

Palabras clave: escuela, subjetividad, aprendizaje, práctica pedagógica, alumno.

\footnotetext{
1 Licenciada en Ciencias de la Educación. Profesora en Ciencias de la Educación. Docente e investigadora de la Universidad Nacional de San Luis. Docente del Instituto Superior "Nuestra Señora del Carmen". Villa Mercedes, San Luis, Argentina. E-mail: guadigarmg@gmail.com.
}

Diálogos Pedagógicas. ISSN en línea: 2524-9274

Año XVI, No 32, octubre 2018-marzo 2019. Pág. 95-105

DOI: http://dx.doi.org/10.22529/dp.2018.16(32)06 / Recibido: 16-02-2018 / Aprobado: 6-09-2018

Artículo publicado bajo Licencia Creative Commons Atribución-NoComercial-SinDerivar. (c) Universidad Católica de Córdoba. 


\begin{abstract}
The aim of this work was to analyse the processes of subjectivity production around the knowledge and its relation in a public school - with urban marginal zone characteristics. This was possible through the school'sleadership team and the teachers' voices when describing their students and the students' parents in the time frame of the results found during an intervention experience carried out by a Universidad Nacional de San Luis research team.

From the analysis of the interviews performed to the school officials and teachers, using a qualitative methodology, the students' meanings and learning conditions were intended to be questioned. As a result, it was interpreted that, in their speeches about how they describe their students well as their own teaching practice, subjectivating processes are stablished linking them to a specialized knowledge. This is done independently to the types of relations established by the students with their own world, surroundings, interests and their own culture; seeing them as "epistemological individuals" and not as a "subject of knowledge".
\end{abstract}

Key words: school, subjectivity, learning, teaching practice, student.

\title{
Introducción
}

Este trabajo se inscribe en el marco del proyecto de investigación: "Escuela, Currículum y Subjetividad: los imaginarios, las prácticas y los vínculos en la producción de subjetividad". PROICO 4-1514. Facultad de Ciencias Humanas. Universidad Nacional de San Luis.

Desde este proyecto se reconoce a la institución escolar en su faceta productora, que habilita a reflexionar sobre las formas de existencia de los sujetos y las modalidades de serlo, en una determinada cultura, producida por ciertas prácticas sociales conformadas como dispositivos (Bozzolo, Lohoste \& Bonano, 2008).

En efecto, durante muchos años se pensó a la escuela como una institución que transmitía, por medio de un conjunto de conocimientos y saberes, e incluso por la misma forma de la relación pedagógica, un compendio general de normas, valores y hábitos de la sociedad. Así los niños abandonaban poco a poco la formación particular de sus familias y paulatinamente accedían, vía la mediación de sus maestros, a la cultura universal y a los desarrollos de las ciencias, lo que los constituía, mediante un proceso de internalización, en ciudadanos. Según esta concepción, la institución escolar era capaz de formar, en una misma acción, actores sociales integrados, sujetos autónomos y críticos, seres racionales y libres. La escuela desarrollaba un proceso institucional transformador de los valores en normas y de las normas en personalidades. Por ello, la educación y la organización escolar debían asegurar simultáneamente la integración social y la promoción individual (Dubet \& Martuccelli, 1998). 
Dubet y Martuccelli además reconocen que perduró durante mucho tiempo una mirada ingenua sobre la institución, ya que identificaba a la socialización, la formación de actores sociales, la subjetivación y la producción de sujetos como una continuación de procesos, en un orden casi espontáneo de las cosas. No obstante, en un momento histórico de caída de los metarrelatos de la modernidad, donde las viejas instituciones de la sociedad y la cultura se han vuelto "fragmentos", la escuela hoy se ve desfondada.

Testificar el presente, a partir de advertir el quiebre en el lazo social escuelacomunidad, no es solo de lo que se ocupa este trabajo, sino también de pensar en esos procesos de subjetivación que, una vez producidos, no necesariamente implican un salto al vacío, sino que permiten el despliegue de nuevas formas de vinculación $y$, por ende, nuevos procesos de subjetivación.

Razones que hoy interpelan y llevan a seguir preguntándose acerca de ¿cuáles son los procesos de subjetivación que hoy se construyen en la escuela y, en particular, en la escuela en la que se llevó a cabo la investigación?

Se asume que es una pregunta que hoy atraviesa y ahonda múltiples dimensiones, siendo sus respuestas escurridizas y singulares; no obstante, se cree que el ejercicio de realizarla aporta una mirada más cercana de los efectos que pueden estar operando en los sujetos-alumnos de esta escuela.

Desde esa interpelación es que se articula este trabajo, en cuanto se indaga sobre los procesos de producción de subjetividad de alumnos insertos en una escuela de gestión estatal -con características de urbano marginal- a través de las voces de sus directivos y docentes.

Como eje analizador se asume el lugar de la escuela como posibilitadora de sentido en relación al saber, para ello se toman algunos de los conceptos del autor Bernard Charlot en cuanto repiensa "la relación con el saber", ya que se considera que los interrogantes que plantea permiten mirar a la educación como una mediación en la constitución del sujeto; por lo que, "educar (se) es constituirse en sujeto" (2008, p. 149).

\section{Consideraciones metodológicas}

A modo de presentación, la escuela seleccionada cuenta con una matrícula que ronda los 700 alumnos, niños y niñas de 4 a 18 años. Posee nivel inicial, primario y ciclo básico del secundario. Los alumnos provienen de familias nacidas de países vecinos como Chile y Bolivia; además un gran porcentaje es procedente de provincias como Mendoza, San Juan y Jujuy. Según la descripción del Proyecto Educativo Institucional (PEI), las condiciones de estos grupos de alumnos, en un alto porcentaje, implican tener dificultades en el aprendizaje debido a diferentes situaciones: desnutrición, mala alimentación, abandono, familias mal constituidas, problemas de adicciones, violencias, baja autoestima, entre otros.

La metodología construida para esta experiencia de indagación se decidió desde un encuadre cualitativo, con fines inicialmente exploratorios, y, 
posteriormente, de intervención en la realidad. Desde allí se propuso realizar un abordaje de la institución, que tuvo en una primera etapa una serie de encuentros previos con los directivos, charlas informales con distintos actores institucionales y recorridos por los espacios institucionales, para lograr aproximarse a la vida cotidiana de la escuela.

En una segunda etapa se armaron protocolos y se realizaron entrevistas a directivos y docentes, de los resultados es que se construyeron estos análisis y reflexiones.

\section{Consideraciones conceptuales}

Bernard Charlot en 1997, en la Universidad de París 8, con el equipo ESCOL (Escuela, Educación y Colectividades Locales), crea una línea de investigación sobre la relación con el saber, con el objeto de comprender la experiencia escolar de los jóvenes de origen popular en Francia. Inicialmente su propósito fue reflexionar y comprender la producción y la reproducción de las desigualdades sociales en la escuela. Charlot desarrolla la idea de relación con el saber, a la que define como "el conjunto de imágenes, expectativas, juicios relacionados a la vez con el sentido y la función social del saber y de la escuela, con la disciplina enseñada, con la sustitución de aprendizajes y con uno mismo" (Charlot, 1997, p. 90).

La afirmación "la relación con" permite enunciar una configuración sin exponer sus términos. La relación con el saber no designa el saber, sino un vínculo de un sujeto con un objeto. El empleo de la expresión en singular, lo mismo que el uso del artículo definido en lugar del indefinido, refuerza el aspecto de la esencia de la relación (rapport) con el saber.

Es importante señalar que tres teorías forjan el fundamento teórico de esta relación con el saber: la del sujeto, la del sentido y la del saber, entendida convencionalmente como "RAS". En la teoría del sujeto (individual o colectivo) la relación con el saber está vinculada a la necesidad de analizar su situación, su posición, su práctica y su historia, a fin de darle su sentido propio.

La relación con el saber se convierte entonces en la creación permanente de saber sobre sí mismo y sobre lo real; se convierte en un proceso creador de saber, mediante el cual un sujeto integra todos los saberes disponibles y posibles de la época. (Beillerot, Blanchard-Laville \& Mosconi, 1998, p. 66)

Por lo tanto, se hace referencia a la "relación con el saber", en cuanto proceso dinámico, y al vínculo entre saber y psiquismo de un sujeto. A través de los saberes, el sujeto intenta un cierto dominio del mundo en el que vive y se comunica con otros sujetos y consigo mismo. La relación con el saber es de carácter singular, particular e íntimo del sujeto en un contexto social y en un momento histórico dado. Se vincula al sujeto con un objeto que es "el saber" y que no remite a conocimientos o saberes específicos, sino a una globalidad.

La relación con el saber de un sujeto se desarrolla en una cultura, una institución, un grupo social o una familia, que determina una orientación en vez de 
otra $y$, en este sentido, atestigua su historia. Al aprender, "el sujeto forma un relato", forma "su saber" a través de la reorganización permanente de su psiquismo, es decir, de una conciencia y de un inconsciente. Se concibe como proceso dinámico, que implica a su vez una estabilidad que puede traducirse en fenómenos lo suficientemente repetidos como para ser observados (Beillerot, Blanchard-Laville \& Mosconi, 1998).

Desde esta teoría se rompe con ideales homogeneizadores que provienen del convencimiento de que existe una génesis inicial de igualdad entre los sujetos, ya que dos sujetos nunca aprenden exactamente lo mismo, puesto que cada uno crea el saber, lo fabrica, lo produce, lo inventa.

El sujeto no sabe qué sabe hasta el momento de su aplicación, de su puesta en actividad: "En toda unidad de saber, el aprendizaje no es registro, sino aprendizaje de una relación con ese saber, que incluye, para cada sujeto, los imaginarios de ese saber, sus usos, etc." (Beillerot, Blanchard-Laville \& Mosconi. 1998, p. 67).

Para Charlot es el sujeto el que aprende (nadie puede hacerlo en su lugar) pero él solo puede aprender por la mediación de otro (frente a frente o indirectamente) y participando de una actividad. Esa actividad y el objeto, respecto sobre el cual dice presentan especificidad, debe ser tomada en cuenta para comprender la relación con el saber y aún más para comprender las relaciones con los saberes.

La educación es una mediación en la constitución del sujeto y no solo posición, acción, actividad, subjetivación, habitus. La educación es, fundamentalmente, el triple proceso por el cual, de manera indisociable, el hijo del hombre se vuelve humano, miembro de una sociedad, y de una cultura en un lugar y en un momento dado, un sujeto con su historia personal. Es el movimiento de humanización, de socialización, de subjetivación; es cultura, como movimiento de construcción de sí mismo, es derecho al sentido, a las raíces, a un futuro: es derecho a lo universal, a la diferencia cultural, a la originalidad personal (Charlot, 2008, p. 149).

Para entender esta teoría es necesario comprender lo que significa el aprendizaje y sus figuras, hay que distinguir entre la relación con el aprendizaje (forma más general) y la actividad-acción-relación al saber (forma específica de la relación -rapport- al aprender).

Analizar la relación con el saber es estudiar al sujeto confrontado con la obligación de aprender, en un mundo que comparte con otros, la relación con el saber es la relación con el mundo, consigo mismo y con otros. Analizar la relación con el saber es analizar una relación simbólica, activa y temporal (Charlot, 1997, p. 89).

Así, entonces, la cuestión del aprender es más amplia que la del saber, en dos sentidos. Primero, hay una manera de aprender, como el caso del especialista de un conocimiento, que es el mejor ejemplo del sujeto epistémico, que no consiste en apropiarse de un saber en el sentido de un contenido de pensamiento. Segundo, en el momento mismo en que se busca adquirir un tipo de saber, se establecen 
otros tipos de relación con el mundo. Este segundo aspecto implica hablar de "un sujeto del saber" y no de "un sujeto epistémico".

El "sujeto en relación con el saber" es visto por la filosofía clásica en relación con la razón, el espíritu, el entendimiento y sus facultades de aprehender lo que allí circula, acontece, tiene lugar. El "sujeto del saber epistémico" se sitúa en una región de conocimiento y excluye otras. El laboratorio es, en este sentido, un modo de separación total del mundo, las disciplinas escolares también. Por este motivo, la comprensión del "sujeto de saber" debe situarse en las relaciones con el mundo y no con un sistema cerrado y arbitrario, independiente de todo lo que acontece en el mundo. Esto exige conocer la constitución de los saberes diferenciándolos del conocimiento y de la información.

En las instituciones educativas en general, "Ios saberes escolarizados" se transmiten al niño independientemente de los modos de relación que él establezca con el mundo, se desconoce el valor del saber y del sujeto como "sujeto singular", como proveedor de sentido (consciente o inconscientemente) al mundo, a los otros y a sí mismo (Charlot, 2008, p. 41).

Por tanto, los modos de abordaje singulares es lo que se debe investigar, para poder acercarse a desentrañar lo que hoy se plantean como problemáticas del aprendizaje, advertir estas singularidades es lo que podría dar luz a tanta oscuridad e incertidumbre.

En la actualidad, las lógicas capitalistas contemporáneas con la globalización del mercado y del dinero no distinguen las diferencias culturales, porque no piensan en términos de cultura sino en términos de dinero, por lo que este se está convirtiendo en "el equivalente universal no solo del trabajo sino también del saber y de toda producción simbólica" (Charlot, 2008, p. 143).

Para Charlot, se habla de una sociedad de la información y no del saber, pero aclara que no son la misma cosa: "La información es un enunciado de un hecho, el saber comienza cuando el conocimiento de ese hecho produce sentido sobre el mundo, sobre la vida, sobre los otros, sobre sí mismos" (2008, p. 144).

De ahí reflexionamos en la importancia de construir saberes y no solo transmitir información, mediar con los alumnos en relaciones con el mundo, con la vida humana, consigo mismo y con los otros.

\section{Análisis de la información}

Teniendo en cuenta principalmente las entrevistas realizadas a directivos y docentes, $^{3}$ se presentan a continuación las categorías elaboradas para el análisis y algunas expresiones de los entrevistados que permite ilustrarlas.

\footnotetext{
3 Se utiliza la "D" para nombrar a la directora de la escuela y la "d" para señalar a las cuatro docentes entrevistadas.
} 


\section{Modo de entender la función de la escuela desde su mito fundacional}

D: "El proyecto fundacional era acoger a todos los chicos hijos de inmigrantes, de familias golondrinas (...) en un primer momento inmigrantes de Chile, ahora muchos de Bolivia".

d1: "En el origen de la escuela había tres aulas, todo patio. Una señora mayor servía mate cocido a los chicos, los docentes también colaboraban en esa tarea, los papás eran muy colaboradores".

d2: "Los primeros años eran de mucha necesidad, pero fueron años de crecimiento. La gente no tenía nada pero tenía mucha ambición, el padre estaba decidido a llevar al niño a la escuela".

d3: "Los padres colaboraban, éramos una familia. Había compromiso, vocación y ganas de trascender".

Sus sentidos, valores y misión se establecieron en los comienzos y se recuerdan como una etapa ideal, que sella un contrato escuela-comunidad en la que la primera adopta el papel de "acogedora", "comprometida con la realidad de la comunidad", que colabora en todos sus aspectos con ella y que recibe a cambio de la "segunda" el compromiso y la respuesta de los padres. Por su parte, los docentes asumen también esta función en los que se sienten pertenecientes, "como una familia" en la que es clave su colaboración.

\section{Mirada negativa de directivos y docentes en relación de alumnos y padres de la comunidad educativa actual}

D: "Gente de acá de alrededor que era gente de campo, sin educación, sin acceso a la ciudad".

d1: "Las mamás son muy humildes y respetuosas, están más presentes que los papás".

d2: "Acá siempre había delincuentes, ladrones, pasaba algo allá y ¿quiénes eran?, los de este barrio (...) Entonces hemos quedado con ese rótulo".

d3: "Sabemos que no podemos esperar cosas de las familias, no podemos pedirles peras al olmo".

D: "Porque también los padres tienen esa, resuelven todo con violencia, no está la palabra de por medio".

En estas miradas se dan ciertas diferencias, no obstante prevalece una mirada negativa, reprobatoria, que pone el acento en las carencias de los alumnos y miembros de la comunidad educativa actual.

Las formas de hablar, por parte del directivo y de los docentes, de los sujetos "alumnos" y "padres" actuales, desde la carencia, genera un tipo de vínculo en el que los docentes son "dadores" y los alumnos y padres son "seres necesitados e incompletos", lo que actualiza la alianza escuela-comunidad anteriormente descripta. 
d2: "Los chicos no estudian en la casa, estudian en la escuela (...), se nos sugiere que no le mandemos tarea a la casa porque la gran mayoría no la va a hacer, están solitos en la casa".

A propósito de estas miradas, Bernard Charlot (2008, pp. 19-20) las considera como una lectura negativa de los sujetos al afirmar: "Cuando se habla en términos de carencia, epistemológicamente no se produce el menor sentido" El autor sostiene que las carencias no son ficciones, no obstante reflexiona que, "para entender por qué no construyeron competencias, se debe explicar lo que aconteció y no lo que faltó".

El análisis de estos modos de vinculación, tanto en las etapas iniciales de la escuela como en la actualidad, interpela la capacidad de correrse de estos lugares tradicionales, de lo asistencial para poder conocer qué "otras cosas son y hacen" estos sujetos, e invita a pensar en los procesos culturales en los que viven y producen.

\section{Los objetivos pedagógicos que se proponen para trabajar con sus alumnos}

d1: "Que sepan que ellos pueden, que se superen, sean cada día mejores, que estudien, apunten a más, a llegar a la universidad".

d1: "El proyecto actual de la escuela apunta centralmente a la lectoescritura. Todo lo que se trabaja enfoca en esto, en todas las áreas. Es lo que más les cuesta."

d4: "Salir a la comunidad, al barrio. Fuimos a la radio, a la Policía, a la Casa de Gobierno, a leerle a la gente. Tratamos de romper el mito ese de los que son normales y los que no".

D: "La escuela tiene que educar y si los chicos no alcanzan los contenidos es dejarlos a la buena de Dios. Esto, más allá de que asimile las normas de comportamiento, de que cambie en lo posible la calidad de vida".

d2: "Sacarlos, que tengan nuevas experiencias, que vean otras realidades (...)".

d3: "Yo siempre quise darles a estos niños la posibilidad de tener un espíritu crítico y armas para defenderse contra cualquier avasallamiento".

En los objetivos pedagógicos planteados, lo que acontece principalmente es la necesidad de "dar, de sacar, mejorar, capacitar, controlar, la vida de los alumnos y de sus padres o comunidad". Se espera y se hacen muchos esfuerzos para que los alumnos puedan cumplir con estos objetivos. Se plasma la construcción del sentido por parte de los docentes, en cuanto se delimita un perfil de alumno, un tipo de vida y de futuro para estos pero se desdibuja la de los alumnos y de los padres. Entendemos, como plantea Charlot, que "la cultura es una construcción de sentido que permite tomar conciencia de sus relaciones con el mundo, con los otros y consigo mismos" (2008, p. 141).

La cultura expresa y explicita lo que se vive en una comunidad, por lo que surge cuestionarse por los sentidos construidos por los propios alumnos y cuál es el lugar de los docentes y su escuela en estos sentidos. 


\section{Algunas prácticas pedagógicas que desarrollan}

\section{Creación del grado acelerador}

El grado acelerador es una práctica pedagógica que implementan los docentes, buscando responder a una problemática que se genera en la escuela con los alumnos que repiten sucesivamente y en la que se da un desfasaje de edades con el grupo total de alumnos. La propuesta contempla dividir el año en dos cuatrimestres y dar contenidos en un ritmo "acelerado", permitiendo al alumno que en un cuatrimestre curse hasta dos grados.

Según lo expresado por la directora: "Los grados aceleradores funcionan con maestras de grado, con chicos que están con sobre edad, con el objetivo de lograr descenderla. Ya sea en el primer ciclo como en el segundo ciclo, entonces la seño lo que hace es tratar de dar los contenidos mínimos en los grados que ya deberían promocionar, para luego insertarlos al nivel secundario. $Y$ hemos tenido buenos resultados (...), este es el $3^{\circ}$ año que lo implementamos. Los niños que han pasado por el grado acelerador son niños que no han repetido en el nivel secundario. Porque una de las cosas que pensamos que podíamos tener como desventaja era que al tener solamente lo mínimo nos podían faltar más cosas para el secundario $y$, bueno, hacer que el niño repitiera, por ejemplo, primer año. No nos ha pasado eso con los que (...) sí se han llevado materias a rendir, pero ya algunos ya terminaron el $3^{\circ}$ año, o sea, ya están en otra escuela".

Esta práctica pedagógica implementada contempla un espacio grupal de mayor paridad en edad para los alumnos repitentes, lo cual se considera puede operar positivamente en estos; no obstante, se advierte una mirada acerca del conocimiento en términos acumulativos y en la que el proceso de aprendizaje se "acelera" para permitir la promoción. Se plantea la enseñanza de "lo mínimo". El lugar en relación al saber y lo que les sucede a los alumnos en torno a este parece desdibujarse en la promesa de lograr pasar de grado exitosamente.

\section{Inclusión de alumnos con parálisis cerebral}

La incorporación de los alumnos con parálisis cerebral es una práctica pedagógica que implementaron los docentes, buscando responder a una de las problemáticas de la inclusión que condicionan a la escuela actual y para las cuales se hace necesario encontrar respuestas colectivas y políticas públicas.

D: "En el Nivel Inicial, trabajan muy bien las chicas (...) y, bueno, de hecho ellas fueron las primeras que aceptaron dos chicos con parálisis cerebral, en silla de ruedas. En segundo ciclo están ahora los chicos y ellos empezaron en Nivel Inicial. O sea, las chicas fueron las primeras que dieron el sí, de poder tenerlos más allá de todas las dificultades que tuvimos, porque fueron un montón. Trabajaron con ellas primero, después con las chicas de la tarde de Sala de 5 (pasaron todos al primer ciclo) y ahora los chicos están en el $2^{\circ}$ ciclo. En $2^{\circ}$ ciclo costó un poquito más con las maestras. Pero, ahí vamos, adaptándose".

La inclusión de alumnos con parálisis cerebral en la escuela es presentada por la directora con orgullo. Sin dudas implicó, para la institución, superar varios obs- 
táculos, principalmente, con los docentes de los diferentes niveles. No obstante, esto interpela a comprender cómo la directora y los docentes significan esta práctica de inclusión en la escuela. ¿Qué sucede con los alumnos y su relación con el mundo, y con el aprendizaje principalmente, en una escuela cargada de tareas y de funciones, que intenta abordar diversas problemáticas, priorizando tal vez el incluir, más allá de lo que suceda en torno a la relación con el saber de los sujetos que están en estas condiciones?

\section{Algunas conclusiones en torno a los sujetos del saber}

En función de todos estos aspectos analizados, con respecto al modo en que los docentes y los directores entienden su tarea en la escuela, en relación a sus alumnos y los procesos de subjetivación que construyen, se entiende que opera el mito fundacional de la institución, que configura un modo de vinculación y un tipo de escuela que se concretizan en las prácticas pedagógicas que se proponen y en sus objetivos pedagógicos. Este mito originario instala al sistema institucional en un tiempo circular, que en las distintas etapas se va resignificando de nuevos sentidos en relación al originario. Lo educativo se liga a lo asistencial, eso constituye el arquetipo subjetivo de ser docente, que toma como elemento identificativo "el ser dador, asistencial, colaborador". Se instala, siempre desde el plano de la subjetividad, una misión mesiánica que reproduce o refuerza un tipo de relación con el saber.

De las representaciones que se describen en relación al trabajo pedagógico, se puede interpretar que responden a esta "alianza originaria" o "mito originario" que sitúa a la escuela como dadora, con una fuerte función asistencial; y a los alumnos y a la comunidad educativa, como beneficiaria de esa asistencia, ya que se los considera "carentes" o excluidos de la sociedad en general.

Desde la operación del mito fundacional y de la mirada negativa de los sujetos de los alumnos y de su comunidad es que se establecen prácticas educativas que vienen a dar resultados efectivos, desde una lógica organizacional que permite que las cosas sucedan. Se gestionan los espacios y los tiempos en prácticas educativas, como la creación de "un grado acelerador" y la inclusión de alumnos con parálisis cerebral que describe la directora.

Establecida así la relación de los directivos y los docentes con los alumnos y sus padres se puede pensar que primordialmente se coloca a los alumnos en un lugar de "sujeto epistémico", en el que se lo piensa desde la vinculación con un conocimiento especializado, se sitúa en una región de conocimiento y excluye otras, lo que es un modo de separación del mundo desde las disciplinas escolares. Los contenidos de la escuela se transmiten a los alumnos independientemente de los modos de relación que ellos establezcan con el mundo. Así, se plantean objetivos pedagógicos como "centrarse en la lectoescritura" o desear que "lleguen a la universidad".

Al dilucidar con mayor claridad, el posicionar a los alumnos desde un "sujeto epistémico" a un "sujeto del saber" plantea una diferencia, ya que a este último 
se lo piensa en las relaciones con el mundo y su entorno, con sus intereses y su propia cultura. Al "sujeto del saber", se lo piensa con él y no para él. Se construyen sentidos y no se los piensan e imponen previamente.

Finalmente se interpreta que se va construyendo un encadenado de sentidos en relación a la función de la escuela y al lugar, por lo tanto, que se le asigna al saber, en esta institución en particular, con este modo de mirar a sus alumnos y a su comunidad, y de entender su propia tarea como educadores, que necesita ser repensada si se buscan realmente procesos transformadores.

Se entiende que pensar en la vinculación de los alumnos con el conocimiento que se enseña en la escuela adquiere valor en cuanto ilumina la lectura de diferentes prácticas educativas que, a veces, se eligen desde las mejores intenciones, pero que reproducen ingenuamente lugares comunes, de anulación del sujeto, de desigualdad y que se alejan de posibilitar una educación como "transformación", "mediación" y "humanización".

Se concluye en la necesidad de interpelar constantemente los sentidos y las condiciones del aprendizaje, teniendo en cuenta los intereses singulares y los más universales, al develar, a su vez, el lugar de los docentes como sujetos del saber, en cuanto que un docente que no puede pensar su vinculación con el saber ni los sentidos que construye no puede pensar en los sentidos y saberes que construye con sus alumnos.

\section{Referencias bibliográficas}

Beillerot, J., Blanchard-Laville, C. \& Mosconi, N. (1998). Saber y relación con el saber. Buenos Aires: Paidós.

Bozzolo R., Lohoste M. \& Bonano, O. (2008). El oficio de intervenir. Políticas de subjetivación en grupos e instituciones. Buenos Aires: Biblos.

Charlot, B. (1997). La relación con el saber. Elementos para una teoría. Montevideo: Trilce.

Charlot, B. (2008). La relación con el saber, formación de maestros y profesores, educación y globalización. Montevideo: Trilce.

Dubet, F. \& Martuccelli, D. (1998). En la escuela. Sociología de la experiencia escolar. Buenos Aires: Losada. 